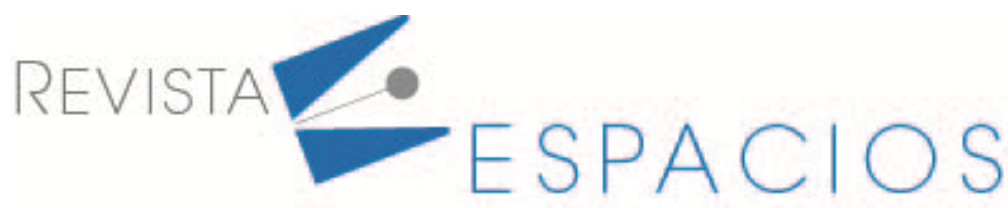

Vol. 42 (06) $2021 \cdot$ Art. 6

\title{
Medición de indicadores para analizar el impacto económico y social de las Cooperativas de Ahorro y Crédito
}

\section{Measurement of indicators to analyze the economic and social impact of the Savings and Credit Cooperatives}

SIMANCA, Fredys Alberto ${ }^{1}$

BLANCO, Fabian ${ }^{2}$

PÁEZ, Jaime Alberto ${ }^{3}$

CORTES, Jairo Augusto ${ }^{4}$

Resumen: El artículo socializa el diseño de un software para la validación de unos indicadores para analizar el impacto económico y social de las cooperativas de ahorro y crédito, analizando la información de la organización cooperativa, para validar de esta manera el cumplimiento del objeto social y económico, generando datos relevantes, confiables y útiles, para la toma de decisiones. El software se diseñó bajo la metodología clásica en cascada siendo necesario para las prácticas sociales esenciales de las cooperativas.

Palabras Claves: cooperativas; impacto; social; económico; indicadores.

\begin{abstract}
The article socializes the design of a software for the validation of some indicators to analyze the economic and social impact of the savings and credit cooperatives, analyzing the information of the cooperative organization, to validate in this way the fulfillment of the social and economic purpose, generating relevant, reliable and useful data for decision making. The software was designed under the classic cascade methodology, being necessary for the essential social practices of cooperatives.
\end{abstract}

Keywords: cooperatives; impact; social; economic; indicators.

\section{Introducción}

En respuesta a los distintos inconvenientes económicos del mundo, las cooperativas emergen como un modelo organizacional innovador para crear sistemas económicos basados en valores y principios éticos que den prelación a la complacencia de las necesidades y expectativas de sus asociados (Dávila Ladrón de Guevara, 2005); necesidades éstas que se sitúan por delante de la maximización de la riqueza particular, teniendo en cuenta además lo establecido por Naciones Unidas afirmando que las cooperativas, son un modelo asociativo que complementa los intereses y necesidades de los ciudadanos, capaces de ejercer un proceso de caracterización de los componentes y medidas que tienen impacto en la innovación mundial del desarrollo sostenible (Alvarez, Rodríguez, Sánchez, \& Fajardo, 2016). No obstante, recalcando que el capital es un medio no un fin.

\footnotetext{
${ }^{1}$ Profesor Investigador. Universidad Cooperativa de Colombia. Ingeniería de Sistemas. Colombia. Email: fredys.simanca@campusucc.edu.co

2 Profesor Investigador. Universidad Cooperativa de Colombia. Ingeniería de Sistemas. Colombia. Email: fabian.blanco@campusucc.edu.co

${ }^{3}$ Profesor Investigador. Universidad Cooperativa de Colombia. Ingeniería de Sistemas. Colombia. Email: jaime.paez@campusucc.edu.co

${ }^{4}$ Profesor Investigador. Universidad Cooperativa de Colombia. Ingeniería de Sistemas. Colombia. Email: jairo.cortes@campusucc.edu.co
} 
El cooperativismo tiene a su vez un notable crecimiento a nivel global, dado su enfoque innovador en el ámbito digital, para poder competir con la banca mundial, para el sector es esencial mantener una significativa presencia en varios países a nivel mundial y en especial a países de Europa, entre ellos Alemania, Francia y los Países Bajos.

De otra parte, estas organizaciones, asumen que su avance tecnológico es, en ocasiones, más vertiginoso que el adecuado nivel de aprovechamiento que alcanzan de ellas sus asociados e interesados, se proyecta que pueden lograr un papel significativo en el fomento de una cultura financiera de base innovadora que permita la evolución digital del sector y de la sociedad, con el fin que logre determinar su viabilidad competitiva en este ámbito donde la transformación digital está obteniendo velocidades de interconexión mundial avanzada (Ortiz Ocaña, Paez, \& Cortes, 2019), tanto en el campo financiero como en el conjunto de la sociedad, lo anterior para las cooperativas se está convirtiendo en el punto clave de su destreza de futuro (Palomo Zurdo, Fernandez Torres , \& Gutierrez Fernandez, 2018).

La carga económica de las cooperativas de ahorro y crédito a nivel mundial, si bien es significativa, muchas veces se ve desestimada y en ocasiones totalmente ignorada. Aun así, algunas de las entidades financieras más importantes a nivel mundial, funcionan como cooperativas. Además, el crédito cooperativo asegura que las compañías se mantengan a flote logrando tener importancia la innovación digital (OIT, 2019), tanto en sus métodos de operación interna, como en lo que tienen que ver con la correlación de servicios de acuerdo con su base societaria y de clientes.

Las cooperativas de ahorro y crédito en Colombia han sido un eje fundamental para el desarrollo de la economía del país, teniendo como base la inclusión de societarios con objetivos claros de avanzar y aportar a la sociedad y cuya función principal es la de adelantar actividad financiera exclusivamente con sus asociados, su naturaleza jurídica se rige por las disposiciones de la ley 79 de 1988; se encuentran sometidas al control de la Superintendencia de Economía Solidaria (Duque Montoya, 2011).

Según la información reportada a los entes de vigilancia y control a corte diciembre de 2018, este grupo de entidades atiende las necesidades de financiación, ahorro e inversión de 3.588 .534 colombianos de manera directa, lo cual significa que el impacto a nivel de grupo familiar puede estimarse en 12.5 millones de personas. Esta base social se encuentra compuesta por un $47 \%$ de asociados hombres y un $53 \%$ de asociadas mujeres (Confecoop, 2018).

Es necesario destacar el rol que han asumido las cooperativas de ahorro y crédito en el tema de inclusión financiera, cuya labor justamente ha sido llegar a la base de la pirámide desde hace muchos años y por eso está sumando casi 4 millones 500 mil colombianos asociados a ese tipo de entidades (Confecoop, 2014). Este procedimiento se debe a una mejor gestión de sus dirigentes y al asesoramiento de la Superintendencia, a partir de la implementación de los indicadores de ley y económicos, controla y hace seguimiento a las cooperativas para verificar el desempeño y cumplimiento de los parámetros que deben practicar para su buen funcionamiento (Duque Montoya, 2011). Por otro lado, el hecho que la actividad financiera cooperativa no esté cobijada con la sobretasa transitoria de renta (este es un impuesto a las entidades financieras sobre sus ingresos), genera un mensaje de estímulo y reconocimiento al formidable papel que desempeñan las cooperativas de ahorro y crédito, en políticas de inclusión social, económica y financiera, especialmente en áreas de pequeños ingresos y en mercados de la geografía nacional que no cuentan con servicios bancarios o son atendidos con algunas limitaciones por otros actores financieros (Portafolio, 2019), (García Reyes, Padro Vite, Salazar Cantuñi, \& Mendoza Rodriguez, 2018).

Actualmente existe un creciente interés en medir el impacto y en determinar la viabilidad financiera de los programas de microcrédito. Sin embargo, más allá del consentimiento alcanzado acerca del fin actual de las microfinanzas y de su potencial impacto, las maneras de obtener excelentes resultados, así como la forma de 
evaluarlos, están creando fuertes discusiones en la retórica especializada en este sector. Este tipo de apreciación se reveló como inconveniente para establecer si estas entidades lograban o no su objetivo de disminución de la pobreza. Es por lo que, surge la necesidad de adelantar estudios de evaluación de impacto social y económico (Garcés Rodríguez, 2008).

Basados en lo anterior, surge la inquietud de medir el impacto económico y social de las Cooperativas de Ahorro y Crédito en Colombia, para ello se desarrolló un software para el cargue de la data proporciona y su posterior análisis. Esto con el propósito de evidenciar el impacto de responsabilidad social y económica, según la metodología aplicada y de acuerdo con las políticas de la cooperativa, proponiendo luego un análisis puntual en concordancia con los indicadores generados, con el fin de que a través de los resultados obtenidos, se alcancen soluciones a inconvenientes encontrados y así validar el cumplimiento del objeto social y económico, arrojando un balance social encaminado a informar los resultados empresariales con datos relevantes, confiables y útiles, para la toma de decisiones.

\subsection{Indicadores y medición}

Indicador es la definición a un término cualitativo o cuantitativo observable, que admite describir características, procedimientos o fenómenos del contexto a través del avance de una variable o el establecimiento de una correlación entre variables, dicha variable confrontada con etapas preliminares, productos equivalentes o una meta o compromiso, logra verificar y evaluar el trabajo y su progreso en el tiempo. Por lo general, son fáciles de compilar, altamente relacionados con otros datos y de los cuales se puedan obtener rápidamente definiciones útiles y fidedignas (Mineducación, 2014).

Los indicadores y las políticas aplicadas según la ley a la información económica generada por las Cooperativas de Ahorro y Crédito deben generar herramientas para la toma de decisiones oportuna, facilitar procesos de análisis adecuado para actuar en los diferentes casos de acuerdo con los resultados obtenidos (Alvarez, Narvaez, Erazo, \& Luna, 2020). En lo económico, financiero y contable, la información ofrece altas posibilidades de consolidación, por la armonización que significa las Normas Internacionales de Contabilidad - NIIF (Contaduría General de la Nación, 2009) según corresponda al grupo, la naturaleza se reporta a la Superintendencia de la Economía Solidaria.

El proceso de esta información está en cabeza, principalmente de los preparadores de información financiera, generalmente profesionales de la contaduría encargados de elaborar informes, reflejados exclusivamente en los estados financieros de propósito general.

Para analizar dicha información se planteó la necesidad de desarrollar un software para la implementación de la medición de los indicadores de Impacto económico y social en las cooperativas de Ahorro y Crédito de Colombia, para ello fue importante el análisis, a partir de la información contable suministrada por las entidades cooperativas a través del Sistema Integrado de Captura de la Superintendencia de la Economía Solidaria (SICSES), Departamento Administrativo Nacional de la Economía Solidaria (DANCOOP) y de la información suministrada a través del instrumento de encuesta para obtención de datos diseñado para complementar esta información (Confecoop, 2017).

Teniendo como base lo anterior y los indicadores señalados, se utilizó una metodología de observación de la realidad que infiere comportamientos en la medición del impacto económico y social en las Cooperativas, implementando variables que plantean observar los componentes interpsicológicos, diferenciando entre la interdependencia positiva, la construcción de significado y las relaciones psicosociales, siendo tres dimensiones en el aprendizaje para cooperar con otros, es decir, tres fases no temporales necesarias en la ejecución de un trabajo cooperativo positivo (Gutierrez de Moral, 2009), lo cual permite obtener datos claros, teniendo como objeto de observación los asociados, que fueron inicialmente los beneficiarios directos de los créditos de las 
entidades cooperativas. Esto consintió analizar el movimiento económico de la entidad y su cumplimiento del objeto social, frente a sus asociados, logrando así que la información fuera relevante, confiable y útil para tomar decisiones claras y ajustes a políticas contables orientadas a obtener un resultado del balance social que infiera en el mejoramiento de la actividad cooperativa en el sector de ahorro y crédito y por ende al avance económico y social del país.

\subsection{Modelo de medición}

Se refiere al resultado de acciones que generan nuevos conocimientos es decir aquellos aportes reveladores inherentes al estado del arte de una actividad, involucrando mecanismos de estandarización, que logren reconocer la generación de un nuevo aporte al conocimiento, y que a su vez han sido analizados y admitidos para asociarlos a la discusión científica, a la mejora de las actividades de investigación, y a la innovación tecnológica, con el fin que los resultados de estos indicadores sean fuente de innovación. (Torres Naranjo \& Arias Hernàndez, 2017).

\subsection{Balance Social}

El balance social es un documento de contabilidad que no reemplaza el balance del ejercicio. En este informe se encuentran registros de tipo social y ambiental concernientes a las operaciones de la empresa. Además, este balance se puede acompañar de resultados de tipo financiero, pero no significa que se deba repetir la información contenida en el balance del ejercicio. Nace de un procedimiento mediante el cual se evalúan y se informa a los stakeholder (Partes interesadas) los resultados de los indicadores éticos y sociales pertinentes a los económicos, que miden las particularidades con las que una organización alcanza los objetivos propios a través de un desarrollo sostenible y responsable, definiendo el impacto como "el resultado de los efectos de un proyecto" (Rodríguez Espinosa, 2018).

\section{Caracterización de la información}

No obstante, aun cuando existen normas aplicables a la medición del balance social de las Cooperativas y en especial a las de Ahorro y Crédito, la metodología empleada por cada organización de base puede diferir a tal punto que los resultados finales no pueden ser comparables entre sí, lo que hace que la agregación de la información alcanzada sea mínima. De acuerdo con la necesidad de calcular la gestión social y económica del cooperativismo, se han implementado, a nivel nacional e internacional, diferentes planteamientos y metodologías para lograrlo (Confecoop, 2018). Para el caso las Naciones Unidas (2009) han definido el objeto de las cooperativas, por su especificidad bajo variables generales que a su vez pueden identificarse como indicadores de impacto social, entre ellos están: (Rodríguez Espinosa, 2018).

- Aumento de los ingresos y reducción de la pobreza e informalidad

- Promoción del desarrollo empresarial y la creación de empleo.

- Generar inclusión social en el acceso a servicios (especialmente en zonas de vulnerabilidad y persistentes fallas de mercado y Estado).

Por otra parte, los indicadores que arrojen los medidores según las variables dependen de las particularidades de la cooperativa según su actividad, entre los más generales se pueden determinar los siguientes: (Alfonso Alemán, Rivera Rodríguez, \& Labrador Machín, 2008).

- Familia del trabajador.

- Calidad de los productos o servicios.

- Relaciones con Empresas, Organizaciones, etc.

- Vínculo con instituciones representativas de la comunidad. 
Los principales reportes de estados agregados de cooperativas, los tiene la Superintendencia de la Economía Solidaria, La Unidad Administrativa de Organizaciones Solidarias -UAEOS- y la Confederación de Cooperativas de Colombia -Confecoop- (UAEOS, 2018). La información presentada registra diferencias verificadas con períodos distintos, tanto en el reporte, como en el número de cooperativas existentes en Colombia (Ver cuadro 1). En Colombia, diversas organizaciones han elaborado sus balances sociales, sin embargo, estos no se consolidan como informes periódicos con variables definidas, por ello es sustancial hacer el cruce de información entre entidades, para identificar visiblemente por qué las diferencias y hacer las depuraciones según el caso (Alvarez, Rodríguez, Sánchez, \& Fajardo, 2016).

Cuadro 1

Número de Cooperativas según UAEOS, Supersolidaria y Confecoop

\begin{tabular}{|c|c|c|}
\hline $\begin{array}{c}\text { UAEOS } \\
\mathbf{3 1 / 0 1 / 2 0 1 8}\end{array}$ & $\begin{array}{c}\text { Supersolidaria } \\
\mathbf{3 1 / 1 2 / 2 0 1 7}\end{array}$ & $\begin{array}{c}\text { Confecoop } \\
\mathbf{0 2} / \mathbf{0 8 / 2 0 1 7}\end{array}$ \\
\hline 23.058 & 1.803 & 3.666 \\
\hline
\end{tabular}

Fuente: elaboración propia a partir de (UAEOS, 2018).

Lo anterior muestra, como los resultantes de los reportes de las entidades que procesan información de cooperativas no coinciden entre sí; más bien arrojan resultados distintos, conllevando a ser revelados en debida forma para lograr consistencia en cualquier estudio que se pretenda desarrollar. Por consiguiente, se genera la necesidad de buscar modelos para la medición que contengan indicadores, que enfoquen de manera más detallada los estudios o investigaciones realizadas para lograr un análisis asertivo para la mejora a la toma de decisiones según resultados del balance social, promoviendo así la utilización de herramientas de informática como software para facilitar dicha tarea (Galviz Cataño, Caycedo Sánchez, Simancas Trujillo, Solórzano Movilla, \& Montoya, 2017).

\section{Metodología y aplicación}

Para el desarrollo del software, fue importante el análisis, a partir de la información contable suministrada por las entidades cooperativas a través del SICSES, DANCOOP y de la información suministrada a través del instrumento de encuesta para la obtención de datos diseñado para complementar esta información, se cruzaron datos de fuentes secundarias, y se utilizó una metodología de observación. Los indicadores más relevantes para observar teniendo en cuenta las variables antes mencionadas son los siguientes: (Gutierrez de Moral, 2009).

- Construcción de significado: Argumentación sobre el contenido y objetivos del trabajo cooperativo. Planteamiento de dudas y formulación de preguntas y respuestas.

- Interdependencia positiva: Certificación de responsabilidad individual con compromisos de los asociados, si se pide compromiso a otros miembros. Propuestas con preguntas y respuestas sobre la organización cooperativa.

- Relaciones psicosociales: Fijar expresiones de aprobación, ánimo, agradecimiento, disculpa; diálogo social y comunicación abierta favoreciendo la participación de opiniones.

Adicional a lo anterior, se debe tener en cuenta el discurso y las diferentes fases en la construcción del significado, siendo esto el fundamento del aprendizaje cooperativo teniendo como base los siguientes indicadores: (Gutierrez de Moral, 2009).

- Fase de inicio: Organización del desarrollo de las actividades cooperativas y verificación de las responsabilidades de cada uno de los usuarios. 
- Fase de intercambio de información: aportaciones al grupo con la participación de los miembros.

- Fase de negociación: Discusión de resultados con base en indicadores y que no se limiten a aprobar y sin sustento.

- Fase de aplicación: Evaluación final de los indicadores, permitiendo valorar las actividades realizadas por los miembros confirmando realmente el trabajo cooperativo. (Gutierrez de Moral, 2009).

Con base en la anterior metodología se desarrolló el software para la Medición de Indicadores Económicos y Sociales para las Cooperativas de ahorro y crédito, definiendo según indicadores, las políticas para regular los procedimientos de los individuos y actividades las cuales van estrechamente ligadas con el compromiso social corporativo (Enguix, 2013). Lo anterior permite, además, que las organizaciones acompañen la gestión de responsabilidad social de sus usuarios.

Los indicadores utilizados se obtuvieron con la información suministrada, y se determinaron los siguientes basados en (Rodríguez Espinosa, 2018):

- Número de asociados hombres,

- Número de asociados mujeres,

- Número de asociados menores de 14 años,

- Total, número de asociados de la entidad solidaria,

- Número total de empleados mujeres de la entidad solidaria,

- Número total de empleados hombres de la entidad solidaria,

- Número de mujeres cabezas de familia.

Posteriormente según el análisis de respuestas se obtuvo un diagnóstico, arrojando indicadores que permitieran tomar una decisión acerca de la responsabilidad social de la organización. Lo anterior se desarrollaría con el fin de estimular y facilitar las técnicas de gestión social responsable en el engranaje de las actividades de la organización o en este caso de la cooperativa, el resultado es determinante para ejecutar evaluaciones periódicas que permitan establecer políticas, metas e iniciativas logrando así una evolución constante en los procesos cooperativos, con el fin de que se evolucione hacia la sustentabilidad en el tiempo del organismo cooperativo (Instituto Ethos, 2011). Lo cual seguramente infiere comportamientos en la medición del impacto económico y social en las Cooperativas de Ahorro y crédito en Colombia, así mismo logra que los resultados alcanzados, por la aplicación de esta metodología, den la posibilidad de mirar el Sistema de Balance Social, como una herramienta que no sólo contribuya a fortalecer la proyección social de las cooperativas y valorar sus efectos, sino que marca el sentido de pertenencia de los asociados al aumentar su cooperación en los procesos de gestión de sus propios recursos (Alfonso Alemán, Rivera Rodríguez, \& Labrador Machín, 2008).

La investigación se efectuó tomando algunos de los indicadores más relevantes utilizados para la medición del balance social del sector Cooperativo, determinado por la Unidad Administrativa Especial de Organizaciones Solidarias (Rodríguez Espinosa, 2018).

\subsection{Determinación de la data}

La data obtenida y analizada está conformada por 43 tablas con un total de 175.723.016 registros. Posterior al análisis de dichos datos se procedió a identificar los indicadores económicos y sociales que serían analizados de acuerdo con el objetivo de la medición, el cual es generar un resultado que evidencie el impacto de responsabilidad social y económica que definiría el ajuste en dado caso a las políticas de la cooperativa, proponiendo así un análisis preciso en relación con los indicadores validados para la toma de decisiones, esto con el fin de acertar y direccionar de manera correcta el impacto del balance social y económico. 


\subsection{Indicadores económicos y sociales}

Los indicadores económicos fueron clasificados en dos variables para su mejor análisis, en: Créditos e Inversión en educación. Por otra parte, los indicadores sociales se clasificaron en: Usuarios y Educación (Ver cuadro 2).

Cuadro 2

Indicadores económicos y sociales seleccionados

\begin{tabular}{|c|c|}
\hline Variables & Indicadores \\
\hline \multirow{2}{*}{ Créditos } & Número créditos \\
\hline & Total, créditos \\
\hline \multirow{2}{*}{ Educación } & Monto de recursos desembolsados \\
\hline & Número de beneficiados \\
\hline \multirow{7}{*}{ Usuarios } & Número de asociados hombres \\
\hline & Número de asociados mujeres \\
\hline & Número de asociados menores de 14 años \\
\hline & Total, Número de asociados de la Entidad solidaria \\
\hline & Número total de empleados mujeres de la entidad solidaria \\
\hline & Número total de empleados hombres de la entidad solidaria \\
\hline & Número de mujeres cabezas de familia \\
\hline
\end{tabular}

Fuente: Elaboración propia

\section{Resultados}

La primera actividad realizada en la etapa de resultados fue la realización del filtro de las cooperativas de ahorro y crédito. La data original está conformada por un total de 5.291 registros, de los cuales 181, son Cooperativas de Ahorro y Crédito. Fueron estas 181 cooperativas la base para el trabajo realizado y los resultados obtenidos en el análisis del impacto económico y social de dichas entidades.

Se presenta a continuación algunos de los datos obtenidos de estos indicadores.

\subsection{Indicadores económicos}

En la fig. 1 se detalla el número de créditos por año y el total en efectivo asignados a créditos y en la fig. 2 se lista el monto de los recursos desembolsados para educación y el número de beneficiados.

Figura 1

Créditos asignados entre los años 2014-2018

\begin{tabular}{|l|l|l|}
\hline Años & \# Créditos & Total \\
\hline $2014-2018$ & 7.007 .091 & $\$ 68.602 .228 .548 .777$ \\
\hline
\end{tabular}

Showing 1 to 1 of 1 rows

Fuente: Tomada del software 
Figura 2

Monto de recursos desembolsados 2014-2018

\begin{tabular}{|l|l|l|}
\hline Años & \# Recursos & Total \\
\hline 2014 & 2.250 & $\$ 30.583 .803 .632$ \\
\hline 2015 & 3.124 & $\$ 30.581 .967 .006$ \\
\hline 2016 & 3.335 & $\$ 39.383 .006 .717$ \\
\hline 2017 & 3.907 & $\$ 48.399 .177 .821$ \\
\hline 2018 & 496 & $\$ 25.750 .173 .352$ \\
\hline Showing 1 to 5 of 5 rows & \\
\hline
\end{tabular}

Fuente: Tomado del software

Puede observarse en la fig. 2 el crecimiento de los números de créditos que se han venido dando en los años analizados. Salvo el caso del año 2018, esto se debe a que la información reportada en este año no está completa, tal y como se mencionó previamente, lo cual genera dificultades para la toma de decisiones y falla en el manejo de políticas acertadas en dichos casos.

\subsection{Indicadores sociales}

En la fig. 3, se detallan los indicadores de usuarios que fueron analizados. Y, en la fig. 4 se analizaron en este indicador el número de beneficiados por ayudas en educación.

Figura 3

Indicadores sociales para usuarios 2014-2018

\begin{tabular}{|l|l|}
\hline Criterio & Total \\
\hline Número de asociados hombres & 6.758 .594 \\
\hline Número de asociados mujeres & 7.621 .056 \\
\hline Número de asociados menores de 14 años & 3 \\
\hline Total Número de asociados de la Entidad Solidaria & 14.460 .605 \\
\hline Número total de empleados mujeres de la entidad solidaria & 32.671 \\
\hline Número total de empleados hombres de la entidad solidaria & 16.221 \\
\hline Número de mujeres cabezas de familia & 174.027 \\
\hline Showing 1 to 7 of 7 rows & \\
\hline
\end{tabular}

Fuente: Tomado del software 


\section{Figura 4}

Indicadores sociales de

educación 2014-2018

\begin{tabular}{|l|l|}
\hline Año & \# Beneficiados \\
\hline 2014 & 486.941 \\
\hline 2015 & 537.272 \\
\hline 2016 & 852.053 \\
\hline 2017 & 1.056 .215 \\
\hline 2018 & 469.662 \\
\hline Showing 1 to 5 of 5 rows
\end{tabular}

Fuente: Tomado del software

Como se puede observar en la figura 4, el aumento de asociados a cooperativas de ahorro y crédito ha venido en una tendencia creciente, en respuesta al papel fundamental que han venido desempeñando dichas entidades, sin embargo, se vuelve a generar dificultad en los indicadores de 2018 , toda vez que la información no es completa.

\subsection{Interfaz de la herramienta diseñada}

La herramienta desarrollada se trabajó con el motor de base de datos MySQL, lenguaje de programación PHP, maquetado con HTML5 y CSS, y siguiendo la metodología de desarrollo clasifica de software, como es la metodología en cascada (Pressman, 2010). En las fig. 5, 6 y 7 se muestran algunas evidencias de la interfaz desarrollada. 
Figura 5

Vista de informe de Número

de asociados 2014-2018

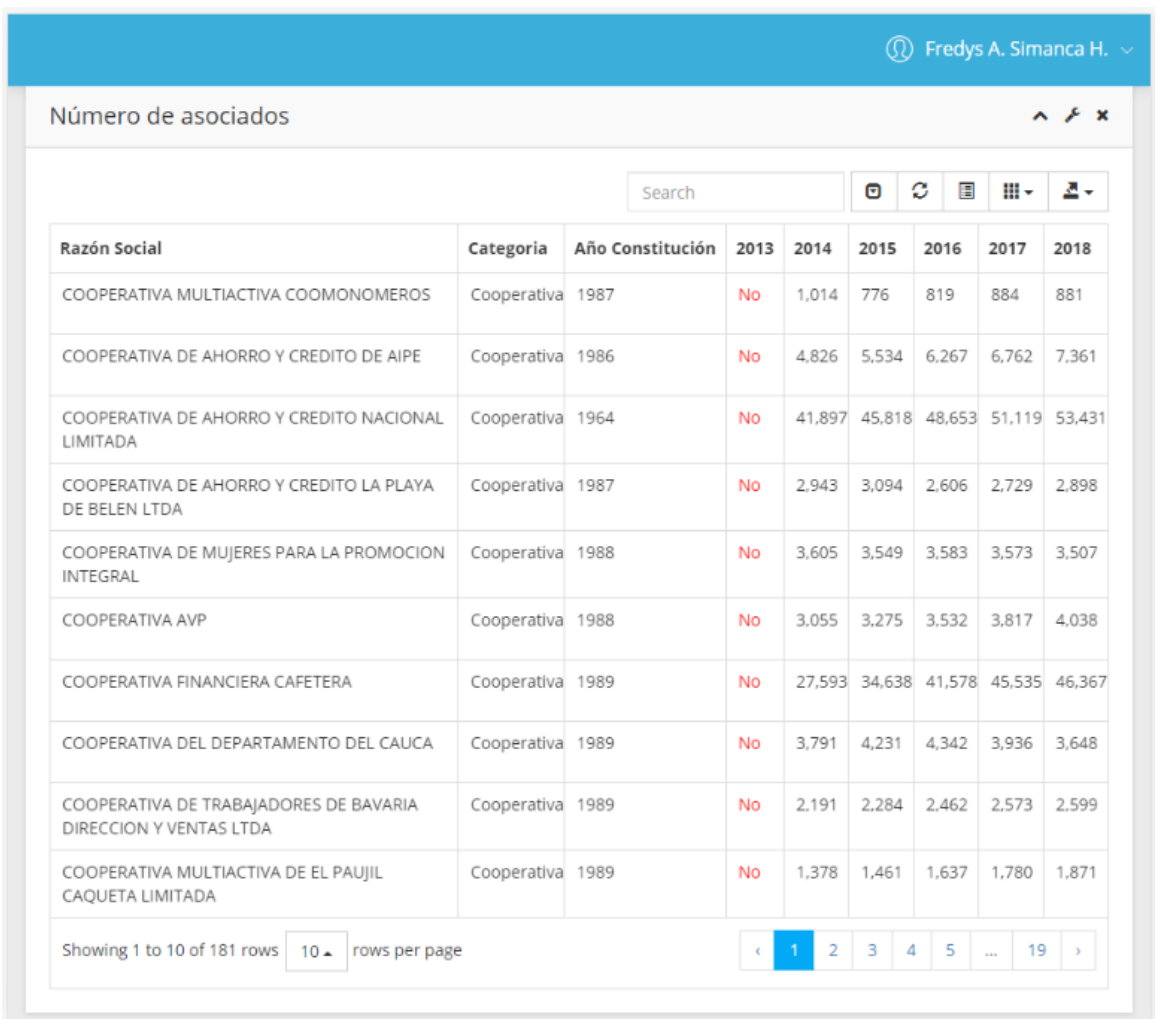

Fuente: Tomado de la herramienta

Figura 6

Vista de informe de Número de empleados 2014-2018

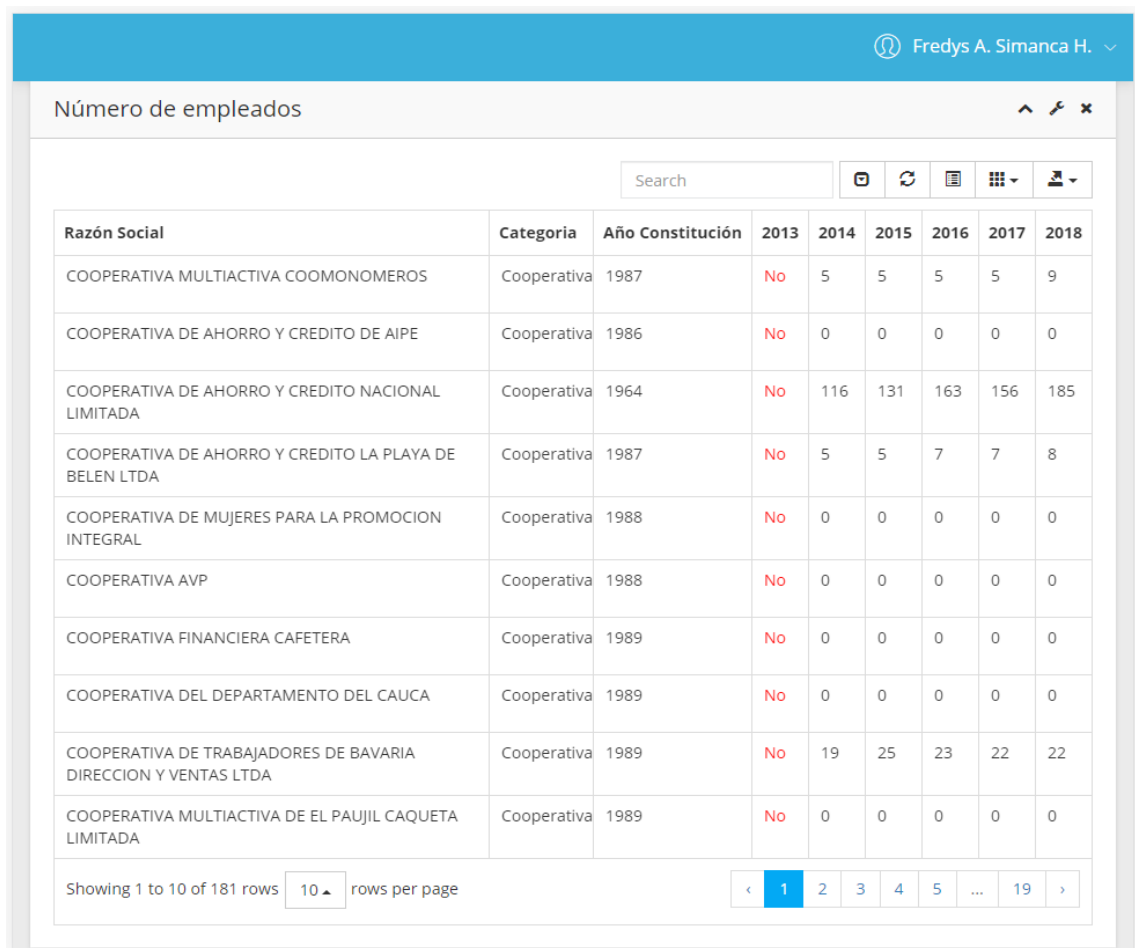

Fuente: Tomado de la herramienta 
Figura 6

Vista de informe Indicadores

sociales de educación 2014-2018

\begin{tabular}{|l|l|}
\hline Año & \# Beneficiados \\
\hline 2014 & 486.941 \\
\hline 2015 & 537.272 \\
\hline 2016 & 852.053 \\
\hline 2017 & 1.056 .215 \\
\hline 2018 & 469.662 \\
\hline Showing 1 to 5 of 5 rows & \\
\hline
\end{tabular}

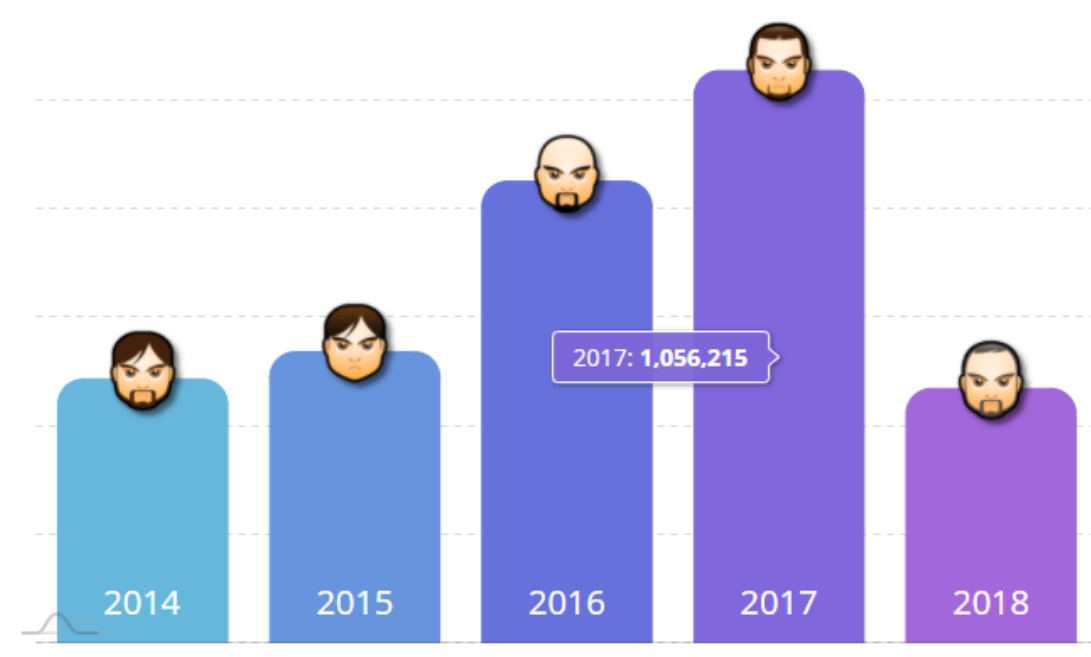

Fuente: Tomado de la herramienta

\section{Conclusiones y recomendaciones}

Luego de los resultados obtenidos de acuerdo a la medición realizada a través del software desarrollado y teniendo en cuenta la clasificación de indicadores utilizados, se pudo verificar que el impacto económico y social que logran las Cooperativas de Crédito y Ahorro en Colombia es muy importante en los últimos años, toda vez que acogen a personas de escasos recursos haciéndolas participe como societarios de estas organizaciones, que no solo les ofrecen beneficios en términos legales, sino también oportunidades económicas, como por ejemplo a madres cabezas de familia, estudiantes y un sinnúmero de individuos.

En la medición a través del software desarrollado permitió analizar el movimiento económico de la entidad y su cumplimiento del objeto social, frente a sus asociados, logrando así que la información fuera relevante, confiable y útil para tomar decisiones claras y ajustar políticas contables orientadas a obtener un resultado del balance social que infiera en el mejoramiento de la actividad cooperativa en el sector de ahorro y crédito.

Según el análisis de respuestas se obtuvo un diagnóstico, arrojando indicadores que permitieran tomar una decisión acerca de la responsabilidad social de la organización, facilitando las técnicas de gestión social responsable en el engranaje de las actividades de la organización o en este caso de la cooperativa, el resultado es determinante para ejecutar evaluaciones periódicas que permitan establecer políticas, metas e iniciativas logrando así una evolución constante en los procesos cooperativos, con el fin de que se evolucione hacia la sustentabilidad en el tiempo del organismo cooperativo (Instituto Ethos, 2011). 
Los resultantes de los reportes de las entidades que procesan información de cooperativas no coinciden entre sí; más bien arrojan resultados distintos, conllevando a ser revelados en debida forma para lograr consistencia en cualquier estudio que se pretenda desarrollar. Por consiguiente, se genera la necesidad de buscar modelos para la medición que contengan indicadores, que enfoquen de manera más detallada los estudios o investigaciones realizadas para lograr un análisis asertivo para la mejora a la toma de decisiones y determinación del impacto social, definiendo el impacto como "el resultado de los efectos de un proyecto.

Teniendo en cuenta que las Cooperativas de Ahorro y Crédito en Colombia han sido un eje fundamental para el desarrollo de la economía del país, y conservando como base la inclusión de societarios con objetivos claros de avanzar y aportar a la sociedad y cuya función principal es la de adelantar actividad financiera exclusivamente con sus asociados, es indispensable propiciar investigaciones que promuevan herramientas de informática, con la capacidad de realizar reportes confiables que permitan analizar la información registrada anualmente por estas organizaciones.

Estos datos obtenidos por los entes de control y otras entidades deben ser capaces de arrojar una información útil, suficiente y real, las fuentes de información deben ser confiables y oportunas, así mismo debe estar sistematizada para facilitar el proceso de análisis, ya que los resultados según las herramientas utilizadas no generan la lectura indicada con respecto al impacto económico y social de los balances sociales, si estos datos no tienen un adecuado análisis (Contaduría General de la Nación, 2009).

\section{Referencias bibliográficas}

Alfonso Alemán, J. L., Rivera Rodríguez, C. A., \& Labrador Machín, O. (2008). Responsabilidad y balance social en las empresas cooperativas. Scielo Revista de Ciencias Sociales, 9-19. Obtenido de http://ve.scielo.org/scielo.php?script=sci_arttext\&pid=S1315-95182008000100002

Alvarez, C., Narvaez, C., Erazo, J., \& Luna, K. (2020). Lógica difusa como herramienta de evaluación del portafolio de inversiones en el sector cooperativo del Ecuador. Revista Espacios, 41(36), 21-38. Obtenido de http://www.revistaespacios.com/a20v41n36/a20v41n36p03.pdf

Alvarez, J. F., Rodríguez, N., Sánchez, C., \& Fajardo, M. (2016). MEDICIÓN DEL IMPACTO SOCIOECONÓMICO DEL COOPERATIVISMO - PROPUESTA PARA SU CÁLCULO. (Instituto de Estudios del Ministerio Publico, \& Unidad Administrativa Especial de Organizaciones S, Edits.) Bogotá D.C., Cundinamarca, Colombia: Instituto de Estudios del Ministerio Público/Unidad Administrativa Especial de Organizaciones Solidarias. Obtenido de https://www.researchgate.net/publication/312520809_Medicion_del_impacto_socioeconomico_del_coo perativismo_propuesta_para_su_calculo

Confecoop. (2014). confecoop.coop. (E. N. Siglo, Editor, E. N. Siglo, Productor, \& El Nuevo Siglo) Obtenido de confecoop.coop: https://confecoop.coop/actualidad/actualidad-2014/co-operatives-united-415/

Confecoop. (2 de 08 de 2017). Congreso Nacional Cooperativo Brindar Herramientas a las cooperativas para asumir los retos del 2018. Recuperado el 22 de 05 de 2018, de Comunicoop No. 2181:

https://preview.mailerlite.com/o9g6n1

Confecoop. (2018). informe de Desempeño 2018. Bogotà D.C.: Confecoop.

Contaduría General de la Nación. (13 de 07 de 2009). LEY 1314 DE 2009-Normas Internacionales de Información Financiera (NIIF). Bogotà D.C., Cundinamarca, Colombia: Contaduria General de la Naciòn. Obtenido de Contaduria.gov.co: https://www.contaduria.gov.co/normas-internacionales-de-informacionfinanciera-niif 
Dávila Ladrón de Guevara, C. (2005). Innovacion y Exito en la Gerencia Cooperativa. Bogotá D.C.: Pontificia Universidad Javeriana.

Duque Montoya, B. (15 de 12 de 2011). Ante falsas cooperativas Alerta por prácticas irregulares. (Enlace Solidario, Ed.) Enlace Solidario, 15, 1-11. Recuperado el 18 de 02 de 2021, de http://www.supersolidaria.gov.co/sites/default/files/public/revistas/pdfs/revista-virtual-15-sep-dic-11.pdf

Enguix, T. (2013). Responsabilidad social en la cadena de valor. Revista PYME AH, 6. Obtenido de http://plataforma.responsable.net/sites/default/files/rs_en_la_cadena_de_valor.pdf

Galviz Cataño, D., Caycedo Sánchez, V., Simancas Trujillo, R., Solórzano Movilla, J., \& Montoya, C. (2017). Los clústers, la tecnología y la academia, una inminente trilogía en las PYMEs de confección de Donmatías, en su proceso de internacionalización. Revista Espacios, 39(11), 8-25. Obtenido de https://www.revistaespacios.com/a18v39n11/a18v39n11p08.pdf

Garcés Rodríguez, C. (2008). Impacto de las Microfinanzas: Resultados de algunos estudios. Enfasis en el sector financiero. Revista Ciencias Estratégicas, 281-298. Obtenido de https://dialnet.unirioja.es/descarga/articulo/2991264.pdf

García Reyes, K., Padro Vite, E., Salazar Cantuñi, R., \& Mendoza Rodriguez, J. (2018). Cooperativas de Ahorro y Crédito del Ecuador y su incidencia. Revista Espacios, 32-38.

Gutierrez de Moral, M. D. (2009). EL TRABAJO COOPERATIVO, SU DISEÑO Y SU EVALUACIÓN. DIFICULTADES Y PROPUESTAS. Univest 09, 1-9. Obtenido de https://dugidoc.udg.edu/bitstream/handle/10256/1956/217.pdf?sequence=1

Instituto Ethos. (2011). Indicadores Ethos de responsabilidad Social Smpresarial. Sao Paulo: Instituto Ethos.

Mineducación. (11 de 06 de 2014). modernizacionsecretarias.gov.co. Obtenido de modernizacionsecretarias.gov.co: https://n9.cl/f9qzn

OIT. (13 de 02 de 2019). ilo.org. (D. Greenfield, Editor, OIT, Productor, \& OIT) Obtenido de ilo.org: https://www.ilo.org/global/about-the-ilo/newsroom/news/WCMS_670577/lang--es/index.htm

Ortiz Ocaña, A., Paez, J., \& Cortes, J. (16 de 12 de 2019). Pymes, competencias laborales y TIC: Competitividad, productividad e innovación organizacional. Bogotá, Cundinamarca, Colombia: Ediciones Universidad Cooperativa de Colombia. Recuperado el 18 de 02 de 2021, de Ediciones.ucc.edu.co: https://ediciones.ucc.edu.co/index.php/ucc/catalog/book/263

Palomo Zurdo, R., Fernandez Torres , Y., \& Gutierrez Fernandez, M. (2018). Banca cooperativa y transformación digital: hacia un nuevo modelo de relación con sus socios y clientes. REVESCO. Revista de Estudios Cooperativos, 161-182. doi:https://doi.org/10.5209/REVE.62490

Portafolio. (18 de 01 de 2019). Portafolio.co. (Portafolio, Editor, \& C. E. Confecoop, Productor) Obtenido de Portafolio.co: https://www.portafolio.co/economia/cooperativas-modelo-para-el-desarrollo-social525358

Pressman, R. S. (2010). Ingeniería del Software: Un enfoque práctico. México F.F.: McGraw Hill.

Rodríguez Espinosa, N. A. (28 de 09 de 2018). Información agregada de cooperativas y sus contribuciones al desarrollo sostenible. España: CIRIEC. Obtenido de Ciriec.es: http://ciriec.es/wpcontent/uploads/2018/09/COMUN-158-TT-RODR\%C3\%8DGUEZ-ESPINOSA.pdf 
Torres Naranjo, M. A., \& Arias Hernàndez, J. S. (2017). Modelo de medición de impacto paralos proyectos sociales de la facultad de Ingeniería en la Universidad Católica de Colombia. Bogotà D.C.: Universidad Católica de Colombia.

UAEOS. (20 de 01 de 2018). Entidades sin ánimo de lucro -Esales- con matrícula activa, por departamento y tipo de organización a 31 de enero 2018. Organizaciones Solidarias, Cundinamarca. Bogotá D.C.:

Organizaciones Solidarias. Recuperado el 25 de 05 de 2018, de

https://www.orgsolidarias.gov.co/node/1192

Esta obra está bajo una Licencia Creative Commons Attribución-NoCommercial 4.0 International

(cc) EY-NO 